\title{
Teaching ESP to university students of International relations with French as their first foreign language considering lexical interference
}

\author{
[Enseignement de l'anglais comme deuxieme langue etrangere a des \\ etudiants des « relations internationales» dont la premiere langue \\ etrangere est le francais compte tenu de l'interference lexicale]
}

\author{
Maximova Olga Borisovna - Spynu Larisa Mikhailovna
}

DOI: 10.18355/XL.2019.12.01XL.13

\begin{abstract}
The article is devoted to English-French lexical interference in teaching English for specific purposes to students of International Relations who study French as their first foreign language. A pilot study was conducted, in which typical lexical errors of Russian-speaking students who study English as their second foreign language were identified. The results of the study indicate that students' typical mistakes are caused by English-French lexical interference. The authors conclude that a special teaching and methodological complex could be effective for students' lexical skills development, their vocabulary expansion and the reduction of errors caused by language interference.
\end{abstract}

Key words: lexical interference, second foreign language, Critical Pedagogy, English language, French language

\section{Résumé}

Cet article est consacré à l' interférence lexicale qui se produit lors de l'enseignement de l'anglais professionnelle aux étudiants des"relations internationales dont le français est la premiere langue étrangère. L'étude pilote a révelé des erreurs typiques des étudiants russophones qui apprennent l'anglais comme la deuxième langue étrangère. L'étude montrent que les erreurs typiques des étudiants sont dues à l'interférence linguistique de l'anglais et du français. Les auteurs concluent que la mise en oeuvre d'un complexe pédagogique et méthodologique spécial dans le processus d'enseignement de la deuxième langue étrangère est effective pour former des compétences lexicales, élargir le vocabulaire et réduire des erreurs.

Mots clés : interférence lexicale, deuxième langue étrangère, pédagogie critique, langue anglaise, langue française

\section{Introduction}

Dans le contexte de la mondialisation, du développement de la communication interculturelle et des contacts internationaux, les exigences relatives aux compétences linguistiques des spécialistes en sciences humaines modernes augmentent et attirent plus d'attention.

L'objectif de réaliser la communication réussie dans le domaine professionnel ainsi que la compétitivité et la mobilité professionnelle obligent les diplômés de maîtriser deux ou plusieurs langues étrangères (Verbitskaya, 2005; Maximova, 2016).

À cet égard, il est important d'étudier la spécificité de l'apprentissage multilinguistique, d'identifier les difficultés rencontrées pendant l'enseignement de la deuxième langue et de définir les moyens visés à les surmonter. À l'heure actuelle, cette problématique attire l'attention des chercheurs, en raison de la situation linguistique actuelle, caractérisée par le développement du plurilinguisme. Ainsi, le phénomène de l'interférence linguistique est activement étudié - "déviations des normes linguistiques qui se produisent dans le discours des multilingues en raison du 
fait qu'ils parlent des langues étrangères, c'est-à-dire à la suite du contact linguistique» (Weinreich, 1953).

Le but de ce travail est d'appliquer la théorie de l'interférence linguistique dans la pratique pédagogique.

\subsection{Principales approches pour l'étude de l'interférence linguistique}

Le phénomène de l'interférence linguistique dans l'étude d'une deuxième langue étrangère (LE2) est généralement considéré, en basant sur deux méthodes : l'analyse de contraste (description comparative des langues afin de prévoir les moments difficiles ou faciles lors de l'apprentissage d'une langue étrangère) et l' analyse des erreurs (examen des problèmes d'interférence post-factum et comparaison des langues pour trouver une source d' erreurs). L'hypothèse de l'analyse contrastive (Ellis, 1986) suppose un transfert positif en raison de la similitude entre les langues et le transfert négatif (interférence linguistique) en raison de la différence linguistique. Malgré certaines restrictions (Choroleeva, 2009), cette théorie est appliquée avec succès par des chercheurs pour prévoir les erreurs potentielles conditionées par le contact linguistique. Dans le cadre de la théorie des erreurs (Corder, 1973, Richards, 1982, Choroleeva, 2009), l'attention des chercheurs est fixée à l'analyse des mécanismes de génération des erreurs et à l'identification des causes de ces erreurs afin de les corriger.

\subsection{Interférence linguistique dans le contexte de la formation multilingue}

Pendant la formation multilingue, il est important non seulement de tenir compte de l'interaction d'une langue étudiée avec la langue maternelle, mais aussi de tenir compte de l'interaction entre différentes langues étudiées. Cette interaction peut créer des difficultés en raison de l'interaction entre les langues, mais elle peut aussi contribuer à l'apprentissage des langues grâce au transfert positif. Ainsi, dans le cas de l'apprentissage d'une deuxième langue étrangère (ci-après, LE 2), il y a un transfert positif des acquis, des savoirs-faire et des compétences acquises lors de l'apprentissage de la première langue étrangère (ci-après LE 1). En outre, le degré de préparation des élèves à l'étude de la LE2 devient plus élevé, car leurs mécanismes de réflexion (mémoire, perception et production de la parole) se forment dans le processus de l'apprentissage et de la maîtrise de la LE1.

Il existe un corpus important de recherches sur le rôle de la langue maternelle dans l'apprentissage de la LE1 et l'interférence linguistique à cette étape (Kuhn, 2007; Sunderman, 2006). Cependant, il y a peu de recherches sur l'interférence qui se produisent dans les conditions de contact de trois langues (langue maternelle, première langue étrangère et deuxième langue étrangère) et des facteurs qui améliorent l'efficacité de l'apprentissage de la deuxième langue étrangère (Duong, 2012; Fusun, 2009; Bello, 2015). En particulier, les questions d'interférence lexicale découlant de l'apprentissage de l'anglais dans le domaine de la communication professionnelle («l'anglais à visée professionnelles») en tant que deuxième langue étrangère sont peu étudiées et analysées.

\section{Principales approches pour la formation des compétences lexicales dans l'apprentissage d'une langue étrangère de la communication professionnelle}

La maîtrise de la terminologie professionnelle est une priorité de l'apprentissage d'une langue étrangère dans le domaine de la communication professionnelle. La compétence lexicale dans le domaine de la communication professionnelle implique la connaissance de la terminologie générale et professionnelle, ainsi que la capacité d'utiliser le vocabulaire acquis dans le discours.

Les critères du succès dans le travail avec le vocabulaire, conformément aux principes fondamentaux des compétences linguistiques du Cadre européen commun de 
référence pour les langues - Apprendre, Enseigner, Évaluer (CECRL) (Conseil de l'Europe, 2011) - est un nombre de mots et d'expressions figées à maîtriser, la maîtrise $\mathrm{du}$ vocabulaire importrant pour la production orale (et écrite), la fréquence de l'utilisation du matériel appris et le contrôle de ce processus ; la connaissance des différences entre le vocabulaire actif et passif, le contrôle des connaissances.

Les exigences citées sont facilement extrapolées au domaine du vocabulaire professionnel.

\subsection{Utilisation de textes compliqués authentiques}

Dans le processus de maitrise du vocabulaire lors de l'apprentissage d'une langue étrangère, toutes sortes de l'activité langagière sont importantes ; cependant, la plupart des chercheurs soulignent le rôle dominant des textes (en particulier écrits) (Gairns, Erdman, 1998). Ceci est dû au fait que c'est le document textuel qui contextualise de nouvelles unités linguistiques ; de plus, les textes sont une source d'informations importantes et motivent les apprenants à mémoriser les mots ; le discours écrit propose le vocabulaire étendu, ce qui favorise l'enrichissement du vocabulaire (Schmitt, 2000).

En outre, il y a plus de possibilités dans le texte cohérent (par rapport aux exercices d'apprentissage) de voir un mot dans un contexte pertinent en liaison avec d'autres unités lexicales, ainsi qu' au niveau des champs lexicaux (Thornbury, 2002). L'analyse de la littérature permet de conclure que le principal moyen d'apprendre le vocabulaire professionnel lors de l'apprentissage d'une langue étrangère doit être présenté dans les textes terminologiques authentiques compliqués.

\subsection{Méthode de lecture intensive}

Le moyen efficace pour former les compétences lexicales dans le domaine de la communication professionnelle peut être, soi-disant, la lecture intensive, ce qui implique un travail minutieux et détaillé des apprenants, sur des textes courts, sous la direction d'un enseignant (Thornbury, 2002).

La lecture intensive contribue à comprendre comment le vocabulaire, la grammaire, la cohérence du texte et son contenu réussissent à atteindre l'objectif communicatif du texte (Nation, 2001). Les apprenants, lors du travail intensif avec le texte, étudient en détail un petit passage ou un texte court de 150-200 mots, tandis que les tâches/les activités qui accompagnent ce texte se composent d'un grand nombre d'exercices lexicaux et de compréhension écrite, dont le volume total dépasse considérablement celui du texte. Les objectifs du travail intensif avec le texte sont les suivants : la compréhension détaillée du texte et l'étude des phénomènes linguistiques à l'exemple du texte, le développement de la pensée linguistique, l'enrichissement du vocabulaire (Nation, 2004).

Les critères généraux de sélection de textes pour la lecture intensive lors de l'apprentissage du vocabulaire sont la valeur du texte du point de vue professionnelle, la conformité du sujet des textes aux intérêts professionnels des étudiants et à leur niveau linguistique (compte tenu de la complexité du sujet, de la logique et de la linguistique), la correspondance à un sujet particulier, la valeur cognitive du texte, la complétude sémantique et la structuration du texte, la représentation dans le corpus des textes d'étude de différents types (descriptif, narratif, argumentaire, informatif, explicatif, explicatif), l'authenticité du matériel de texte d'une variété de genres, de styles scientifiques, journalistiques et pragmatiques. Pendant l'enseignement du vocabulaire professionnel d'une deuxième langue étrangère c'est le caractère interculturel des informations présentées dans le texte qui compte.

\subsection{Application de la méthodologie de la pédagogie critique}

La lecture intensive des textes authentiques compliqués de spécialité, en tant que méthode pédagogique, visant à optimiser l'apprentissage de la terminologie au niveau 
de contact linguistique, s'inscrit parfaitement dans le concept méthodique de la pédagogie critique, qui a été déjà analysée. (Maximova, 2016: 8989-8995).

La pédagogie critique est une approche originale de la pratique d'enseignement, permet aux étudiants de mobiliser leurs compétences de la pensée indépendante, contribuant à la formation des membres actifs, critiques, conscients et responsables de la société (Maximova, 2016: 1003-1010). La spécificité des méthodes de la pédagogie critique est de renoncer aux méthodes rationnelles et dogmatiques de l'apprentissage traditionnel en faveur d'une approche qui tient compte de la diversité des positions et des points de vue des apprenants lors du processus éducatif, de leurs caractéristiques individuelles; cette approche contribue le plus au développement de la compétence professionnelle des apprenants. Dans le cadre de la pédagogie critique, le rôle traditionnel est déconstruit, ce qui implique l'activité de l'enseignant en tant que porteur de connaissances et la passivité de l'étudiant en tant que bénéficiaire, la délégation d'une partie de pouvoirs des enseignants aux étudiants est pratiquée.

Cette pratique permet aux étudiants de s'exprimer et de participer activement à la conception collective des connaissances, et contribue à améliorer leur préparation et leur activité sociales.

Les méthodes de la pédagogie critique contribuent au développement des compétences de lecture et de compréhension écrite des textes étrangers à visée professionnelle, aident à couvrir toute la variété des formes de textes scientifiques modernes, sur papier et sur supports électroniques. En particulier, les résultats de l'étude-pilote ont démontré l'utilité des méthodes de la pédagogie critique dans l'enseignement de la lecture et de la compréhension des textes à visée professionnelle en anglais (Maximova, 2016: 8989-8995).

L'utilité des méthodes de la pédagogie critique dans l'enseignement d'une langue de spécialité est due au fait que c'est dans cet aspect que l'avantage de déléguer les pouvoirs d' un enseignant à un apprenant est le plus manifeste.

En effet, l'enseignant d'une langue étrangère, qui n'est pas suffisamment compétent dans le domaine de la spécialité étudiée par des apprenants, peut jouer un rôle assez organique lors des cours de LS (langue de spécialité).

La possibilité de former l'enseignant (en langue étudiée) à une spécialité donne un intérêt supplémentaire aux cours.

\subsection{Méthodologie de l'enseignement du vocabulaire de l'anglais comme deuxième langue étrangère}

La pratique mondiale a une expérience énorme relative aux techniques d'apprentissage $\mathrm{du}$ vocabulaire de l'anglais comme la LE2 (Wallace, Thornbury). Comme la langue maternelle des apprenants est le russe, le but de notre étude est la métodologie la plus pertinente développée dans le cadre de l'école nationale d'enseignement des langues étrangères, qui repose sur l'utilisation d'un ensemble intégratif d'exercices visé à apprendre le vocabulaire de la LE dans le processus de lecture (Alikina, 2009).

Dans le cadre de cette technique, l'apprentissage du vocabulaire dans le processus de lecture comprend les étapes suivantes :

- étape pré-textuelle : actualisation et présentation associative du lexique pertinent pour la compréhension du texte;

- étape lecture du texte-la perception (repérage) d'un nouveau vocabulaire dans le texte, sa sémantique (formation d'une compétence lexicale réceptive);

- étape traitement du texte informatif (formation de compétences lexicales reproductives et productives);

- étape production orale créative-l'imploi créative libre du vocabulaire dans le discours sur la base d'une bonne compréhension de la forme, de la signification et des caractéristiques fonctionnelles (formation d'une compétence lexicale productive).

XLinguae, Volume 12 Issue 1XL, January 2019, ISSN 1337-8384, eISSN 2453-711X 
Les principales méthodes de travail avec le vocabulaire basées sur le texte à lire comprennent la compréhension et la sélection des unités lexicales, dans le texte, d'une certaine couche de la LE 2 qui appartiennent aux cognates anglais/français; sémantisation de nouveaux mots grâce à des techniques transférées de l'expérience linguistique de la LE 1; écriture de nouveaux mots qui font partie des expressions citées dans le texte; emploie des unités lexicales réceptives dans la production orale lors des exercices «questions - réponses»; effectuer des exercices sur le développement des savoirs-faire et des connaissances lexicaux du texte étudié et des exercices visés à employer les formes modifiées de ce vocabulaire; l'exécution individuelle des exercices pour améliorer la connaissance d'une seule unité lexicale après les activités d'entraînement en employant ces nouveaux mots basés sur ce texte; un groupe d'exercices doit comprendre des consignes sur la comparaison des informations socioculturelles du texte (Alikina, 2009).

\section{Méthodologie de l'apprentissage du vocabulaire professionnel de l'anglais comme deuxième langue étrangère pour les étudiants des "Relations internationales »}

Ayant pour base la méthodologie ci-dessus de l'enseignement/apprentissage du vocabulaire, nous l'avons adaptée aux tâches de l'enseignement de l'anglais comme deuxième étrangère, avec le français LE 1 dans le domaine de la communication professionnelle. De plus, lors de l'élaboration de la méthodologie, l'utilité de la méthode de lecture intensive, en basant sur des textes authentiques compliqués, ainsi que des méthodes de pédagogie critique ont été prises en compte. Ainsi, nous avons élaboré un ensemble d'exercices pour l'apprentissage du vocabulaire professionnel, y compris les exercices d'entrainement de la production orale (PO), exercices préparatifs à la $\mathrm{PO}$ et exercices sur la $\mathrm{PO}$ qui contribuent à la reconnaissance, la perception et la compréhension de différentes unités lexicales du vocabulaire professionnel.

Ce groupe d'exercices fait partie du système d'exercices visant à apprendre la lecture intensive comme un type d'activité de la PO en utilisant des méthodes de la pédagogie critique, et est construit en tenant compte du transfert multilingue, des capacités intellectuelles et linguistiques des apprenants. Dans le cadre de ce groupe d'exercices intégratifs, l'apprentissage du vocabulaire est réalisé en trois étapes.

Première étape (pré-textuelle) est consacrée au travail avec le vocabulaire pour assurer la mémorisation primaire de la compétence lexicale dans le domaine de la communication professionnelle, aux exercices pour former des liens paradigmatiques et syntagmatiques :

a) regroupement de termes-l'activité qui est utilisé pour apprendre aux étudiants d'analyser les termes étudiés et de les savoir combiner en fonction d'un critère commun (signification, structure grammaticale - étymologie) : p.ex. Divisez les termes ci-dessous en groupes selon les critères suivants.

b) repérage de l'intrus - exclure de la liste de mots et d'expressions qui ne sont pas les termes: p.ex. Quels mots et expressions ci-dessous ne sont pas des termes? Pourquoi?

c) comparaison avec les termes du français / du russe selon la structure et la signification: p.ex. Lexique (mots et expressions) cité ci-dessous n'appartient pas à la terminologie? Pourquoi? Comparez le terme anglais avec le terme français /russe correspondant. Quelle est la différence entre leur signification ou leur structure?

Deuxième étape : la formation des compétences lexicales est l'étape de l'entraînement intensif.

Cette étape est combinée avec l'étape de l'étude du texte et le développement des compétences de lecture intensive à l'aide de méthodes de pédagogie critique.

À cette étape, une variété d'exercices oraux et écrits sont travaillés, ce qui permet de développer la capacité d'utiliser des termes, de choisir un terme en fonction de la 
situation, du contexte et la capacité de comprendre tout le texte d'une manière détaillée et avec précision, à savoir :

a) à compléter le texte ou les répliques d'un dialogue - est un exercice générique qui peut être créatif et avoir plusieurs options possibles: p.ex. Complétez le texte par des termes connus ;

b) à répondre aux questions en utilisant le vocabulaire professionnel: p.ex. Répondez aux questions suivantes, utilisez la terminologie professionnelle pour répondre aux questions et respectez le style scientifique de la présentation ;

c) à sélectionner du texte les données les plus intéressantes d'un point de vue professionnel. L'activité visée à encourager les étudiants à analyser l'information obtenue lors de la lecture du texte et du travail avec le vocabulaire professionnel et à décider ce qui est le plus intéressant et important ; à repérer la façon dont les connaissances acquises caractérisent le pays de la langue étudiée : p.ex. Quelle information est la plus intéressante et importante du point de vue professionnel ? Pourquoi?

d) à analyser le texte : p.ex. Sélectionnez dans le texte les mots et expressions relatifs aux lexique politique, sociale, scientifique, les mots de liaison, articulateurs logiques, etc.

Troisième étape du travail avec le texte et le vocabulaire professionnel correspond à la phase post-textuelle, est visée à la mémorisation du lexique et à leur activation dans le processus de la PO et PE (exercices langagiers).

À cette étape, la capacité d'exposer le contenu du texte, d'argumenter est en train de se développer.

Pour développer ces compétences, les activités suivantes sont proposées :

a) La PO sur la situation en utilisant le vocabulaire professionnel. La discussion est proposée d'un point de vue professionnel intéressant, le sujet pertinent sur lequel les étudiants doivent exprimer leur propre opinion en utilisant l'un ou plusieurs des termes proposés : p.ex. Exposez votre point de vue sur le problème en employant les termes proposés;

b) A caractéristiser des événements historiques, des personnalités politiques : p. ex. Quels événements historiques connus pourriez-vous décrire en employant la terminologie proposée? Quels politiciens connus peuvent être caractérisés par le lexique mentionné ? Pourquoi?

c) A préparer un bref exposé en utilisant le vocabulaire professionnel: p.ex. Préparez un petit message. Utilisez les termes proposés;

d) A transférer l'action du texte à la réalité russe - est un exercice pendant lequel les étudiants doivent supposer que la situation décrite dans le texte se passe en Russie: p.ex. Imaginez que l'action du texte se passe en Russie. Qu'est-ce qui aurait pu changer? Écrivez votre version du texte.

Avec les tâches ci-dessus, vous pouvez réaliser des activités visées à travailler et mémoriser la terminologie thématique afin d'éliminer l'interférence lexicale possible. Ces activités peuvent inclure la sélection des synonymes/antonymes, des lacunes à remplir, la traduction bilatérale des unités lexicales dans de différents contextes, des exercices de correspondance de mots, la formation de mots ayant la même racine à l'aide des affixes appropriés.

\section{Recherches expérimentales}

\subsection{Interférence lexicale dans l'étude des termes diplomatiques}

L'accent est mis sur l'interférence lexicale qui se produit lors de l'étude de la terminologie professionnelle anglaise dans le domaine des relations internationales dans le cadre des contacts linguistiques de la LE1 (français) et LE2 (anglais). 
Pour identifier la zone d'interférence lexicale la plus probable, nous avons analysé le «Dictionnaire des termes diplomatiques", qui comprend plus de 1300 mots et expressions (Berridge, Jones, 2003).

L'analyse a montré que plus de $80 \%$ des termes diplomatiques du Dictionnaire sont d'origine française et latine (à comparer : le vocabulaire de l'anglais général contient $38 \%$ du vocabulaire d'origine française) (LeBlanc, Segun). Ce fait donne une importance supplémentaire à la prise en compte de l'interférence linguistique à ce niveau de l'enseignement de l'anglais aux apprenants du FLE comme LE 1.

Ensuite nous avons supposé que l'interférence linguistique se manifesterait différemment selon le degré d'assimilation du terme. Par conséquent, les termes francophones ont été divisé en trois groupes: entièrement assimilé, partiellement assimilé et non assimilé (Maximova, 2016). En basant sur l'expérience pédagogique, nous avons supposé que seuls les termes du premier et du deuxième groupe serviraient de source d'interférence linguistique, alors que les termes non assimilés ne posaient pas de difficultés pour les apprenants du français.

Après avoir sélectionné ce matériel lexical, comme une source évidente de l'interférence linguistique et en s'assurant que les étudiants du FLE comme LE1 font vraiment des erreurs dans ce matériel, nous avons essayé de proposer la méthode pendant laquelle l'effet négatif de l'interférence linguistique est minimisé mais les compétences acquises lors de l'apprentissage du français (transfert positif) sont activées.

\subsection{Hypothèse de la recherche}

En apprenant l'anglais comme LE 2 sur la base du français LE 1, l'interférence linguistique et le transfert positif surgissent; la langue française est une source d'interférence (et de transfert positif) dans l'étude de la terminologie internationale.

Les erreurs typiques dues à l'interférence linguistique de la LE 1 et LE 2 dépendent de l'expérience linguistique de l'étude de LE 1.

Le processus de formation des compétences lexicales dans l'apprentissage de l'anglais comme LE 2 sera plus efficace si l'on élabore une méthode d'apprentissage du vocabulaire professionnel en tenant compte du transfert de la LE 1 des compétences langagières et verbales, des compétences linguistiques et pédagogiques générales en utilisant les techniques méthodologiques de la pédagogie critique, avec l'inclusion dans le processus d'apprentissage d'un ensemble intégré d'exercices d'apprentissage du vocabulaire sur la base de texte authentique de la spécialité « Théorie et Histoire des Relations Internationales » (THRI), sélectionés en tenant compte des capacités des apprenants en mémorisant et perfectionnant le vocabulaire professionnel dans le processus de lecture intensive.

\subsection{Le but de la recherche}

Vérification de l'efficacité de la méthode d'apprentissage du vocabulaire de l'anglais professionnel comme LE2 se fait en tenant compte de l'expérience linguistique des apprenants basée sur l'ensemble d'exercices lexicaux intégrés dans le système d'exercices d'apprentissage de la lecture, en tenant compte du transfert multilingue, en utilisant la méthode de la pédagogie critique.

\subsection{Objectifs de la recherche sont de:}

- montrer que lors de l'étude du vocabulaire professionnel de l'anglais comme LE 2 avec le FLE comme LE 1, l'interférence lexicale et le transfert positif apparaissent ; - identifier les sources d'interférence lexicale et de transfert ;

- analyser les causes des erreurs et trouver la meilleure stratégie pour les corriger ; - élaborer la méthode pour corriger les erreurs dues à l'interférence linguistique anglais / français lors de l'enseignement de la LE de la spécialité "Relations 
Internationales"; tester l'utilité et efficacité des méthodes élaborées à l'aide d'une formation expérimentée.

\subsection{Méthode de recherche}

$\mathrm{Au}$ cours de cette recherche, les étudiants ont été questionnés et testés, les erreurs lexicales typiques des étudiants apprenant l'anglais comme LE 2 ont été identifiées et systématisées, et une méthode pour les corriger a été élaborée et proposée.

La recherche-pilote réalisée confirme l'opportunité et l'efficacité de la méthode proposée d'enseignement du vocabulaire professionnel de l'anglais sur la base de l'ensemble d'exercices lexicaux intégré dans le système d'exercices visés à l'apprentissage de la lecture à l'aide de la méthode de pédagogie critique.

Méthodes utilisées : le questionnaire, l'analyse des dictionnaires et de la littérature pédagogique, le suivi et le contrôle de l' activité éducative, les conversations avec les étudiants, l'apprentissage expérimenté.

\subsection{Participants}

Les participants à cette étude : 40 étudiants de la 3-ème et 4ème année du premier cycle de la faculté des sciences humaines et sociales, specialité «Théorie et histoire des relations internationales » (THRI) qui apprennent l'anglais comme LE 2 dans le cadre du cours «l'Anglais de la communication professionnelle ».

Selon le programme, l'enseignement du cursus de «l'Anglais à des fins professionnelles » se réalisent après la passation par des étudiants d'un cours d'anglais général, lorsque les compétences langagières de base et les compétences générales des apprenants (phonétique, grammaire, formation de mots et communicative) sont déjà formése. Le but du cursus est de mémoriser et développer les compétences langagières de base, ainsi que la maîtrise d'un nouveaux lexique, d'une terminologie spéciale, du style d'affaires et scientifique du discours et des phénomènes grammaticaux et de la formation des mots associés.

Selon le programme d'études, parallèlement à l'apprentissage de la terminologie professionnelle de la LE 2, les étudiants maîtrisent le vocabulaire professionnel de la LE 1 et des disciplines spéciales dans le cadre de leur spécialité principale en langue maternelle.

Deux groupes d'étudiants ont été sélectionnés. La première langue étrangère des étudiants du premier groupe était le français, le deuxième groupe apprenaient la langue orientale (arabe / chinois). L'âge des participants varie de 19 à 22 ans. La sélection des participants a été réalisée en fonction du niveau de maîtrise de l'anglais identifié par les tests, selon les résultats des examens internationaux (DELF - niveau B1) et des questionnaires.

\section{Résultats de l'étude}

\section{1. Étape 1}

Afin de déterminer l'étendue de l'interférence lexicale potentielle des langues en contact, nous avons effectué l'étude comparative de deux langues (anglais et français), puis, par expérience, des cas réels d'interférences - erreurs des étudiants - ont été identifiés.

\subsubsection{Sources d'erreurs lexicales}

Sur la base de la théorie de l'analyse contrastée, nous avons identifié « des points de problème » potentiels qui se posent lors de l'étude de la terminologie diplomatique par les étudiants dont le français LE 1 et l'anglais LE 2: mots-cognates avec sémantique commune et combinaison différente, faux amis du traducteur, acronymes et noms d'organisations internationales, phrase avec "cassé" (sans correspondre aux règles de 
base de l'ordre de mots de la langue anglaise) la place des adjectifs dans la postposition par rapport au mot défini. Ces unités lexicales peuvent être une source de transfert négatif.

Afin de préciser davantage le vocabulaire diplomatique «problématique» d'origine française, l'analyse du Dictionnaire anglais-anglais des termes diplomatiques et des textes pédagogiques a été effectuée.

Pendant le travail nous étions guidés par le fait que les unités lexicales d'origine française (cognates) peuvent être à la fois une source d'interférence et de transfert positif.

En analysant le dictionnaire, nous avons identifié trois catégories de termes diplomatiques - les cognates qui se diffèrent en fonction du degré d'assimilation (totalement assimilé, partiellement assimilé et non assimilé). Dans le même temps, l'analyse de contraste suggère que la source d'erreurs (interférences) ne peut servir que des termes entièrement ou partiellement assimilés (termes de la première et de la deuxième catégorie de notre classification). En ce qui concerne les termes non assimilés d'origine française (termes de la troisième catégorie), ils peuvent au contraire, générer un transfert positif.

L'application de la théorie de l'analyse contrastée (et notre expérience pédagogique) montre que, non seulement les mots de la même racine (cognates) d'origine commune, mais aussi des termes-expressions avec l'ordre de mots "perturbé" peuvent être une source d'erreurs (à savoir, la positon atypique pour l'anglais et typique pour le français, des adjectifs en postposition par rapport au nom, par exemple, " ambassador extraordinary and plenipotentiary »).

Une autre source d'erreurs peut être présentsée par des structures d'attributs binomiaux et polynomiaux (par exemple, career ambassador, World Trade Organization) car ce phénomène linguistique (chaînes attributaires) n'existe pas en français où les prépositions sont traditionnellement un moyen lexical d'expression des relations syntaxiques. L'analyse du dictionnaire permet de mettre en relief des groupes ds termes-expressions: les noms des organisations internationales; les termes diplomatiques formés selon le modèle $(\mathrm{N}+\mathrm{A})$ avec un adjectif en post-position par rapport au mot déterminé.

\subsubsection{Test de détection d'erreurs}

L'étape suivante a été consacrée à l'élaboration d'un test d'identification des erreurs conformément aux "points problématiques" identifiés au cours de l'analyse de contraste.

Le test se composait de trois parties, dont chacune contenait 20 questions «à choix multiples » et des « trous à remplir ».

Chaque partie du test est visée à contrôler et identifier des erreurs potentielles, déterminées par l'analyse du contenu du dictionnaire, dans les trois groupes de termes problématiques: cognates (partie 2), noms d'organisations internationales (partie 1), termes avec adjectif en post-position (partie 3).

Le test de 60 questions «à choix multiples » se composait de trois parties, dont chacune est visée à vérifier un segment spécifique de la maîtrise du vocabulaire professionnel et à identifier les erreurs des étudiants dues à l'interférence linguistique:

1) cigles et acronymes, noms des organisations internationales

2) termes-groupes de mots formés par le modèle " nom + adjectif»,

3) mots dont la forme est similaire mais le sens est diffèrent, c-à-d - termes- cognates, mots à valeurs multiples, faux amis de la traduction et ainsi de suite.

Ces trois segments en tant que sources d'erreurs lexicales potentielles ont été identifiés à la base de l'analyse contrastive, de l'expérience pédagogique de l'auteur, de l'analyse des erreurs dans le processus d'apprentissage, des conversations avec les étudiants, de l'analyse des dictionnaires explicatifs et bilingues, du corpus des textes pédagogiques et des productions écrites des apprenants. 


\subsubsection{Procédure de recherche et discussion des résultats}

Deux groupes d'étudiants A1 et A2 qui apprenaient l'anglais comme LE 2 dont le français était LE1 ont été sélectionnés pour identifier des erreurs lexicales typiques.

Le groupe A1 de 20 étudiants (dont le français ét la LE 1) de la $4^{\text {ème }}$ année (baccalauréat $=$ licence) de l'Université Russe de l'amitié des peuples, faculté des sciences humaines et sociales, specialité Théorie et histoire des relations internationales (THRI) apprenait le français comme LE 1 et l'anglais comme LE 2 ; le groupe A2 de 20 étudiants de la même spécialisation apprenait la langue orientale (chinois / arabe) comme LE1 et et l'anglais comme LE 2.

Les groupes d'étudiants ont été sélectionnés de sorte que leur niveau d'anglais soit le même, ce qui a été déterminé par le questionnaire et par les résultats du teste d'anglais général.

Les deux groupes ont passé le test élaboré par l'auteur Maximova, qui contrôlait les connaissances du vocabulaire professionnel de la spécialité des «relations internationales ».

Les résultats du test du groupe 1 et 2 sont présentés dans le tableau 1 .

\begin{tabular}{|c|c|c|c|c|c|c|}
\hline & Groupe & $\mathrm{N}$ & Moyen & $\begin{array}{l}\text { Std. } \\
\text { Deviation }\end{array}$ & $\mathrm{DF}$ & $\begin{array}{l}\text { T } \\
\text { observé }\end{array}$ \\
\hline \multirow{2}{*}{$\begin{array}{l}\text { Partie 1. (Noms } \\
\text { des } \\
\text { organisations } \\
\text { internationales) }\end{array}$} & $\begin{array}{l}\text { Groupe } \\
\text { A1 }\end{array}$ & 20 & 15,5 & 2,23 & 38 & 1,317 \\
\hline & $\begin{array}{l}\text { Group } \\
\text { A2 }\end{array}$ & 20 & 16,4 & 2,09 & 38 & \\
\hline \multirow[t]{2}{*}{$\begin{array}{l}\text { Partie } \\
\text { (cognates) }\end{array}$} & $\begin{array}{l}\text { Groupe } \\
\text { A1 }\end{array}$ & 20 & 12,2 & 2,12 & 38 & 4,570 \\
\hline & $\begin{array}{l}\text { Groupe } \\
\text { A2 }\end{array}$ & 20 & 15,3 & 2,17 & 38 & \\
\hline \multirow{2}{*}{$\begin{array}{l}\text { Partie } 3 . \\
\text { (expressions } \\
\text { "N+A") }\end{array}$} & $\begin{array}{l}\text { Groupe } \\
\text { A1 }\end{array}$ & 20 & 11,9 & 2,08 & 38 & 1,713 \\
\hline & $\begin{array}{l}\text { Group } \\
\text { A2 }\end{array}$ & 20 & 10,8 & 1,98 & 38 & \\
\hline
\end{tabular}

Tableau 1: Résultats du test de vocabulaire professionnel des étudiants en fonction de la première langue étrangère

Le tableau 1 présente les résultats de l'application des statistiques-T aux valeurs moyennes des résultats du test obtenus par les groupes A1 et A2 lors du travail avec des parties du texte. La possibilité d'appliquer le critère $\mathrm{T}$ de l'étudiant est due à l'homogénéité des variances, confirmée dans tous les cas par l'application des statistiques $\mathrm{F}$ aux écarts-types au niveau standard de valeur $\alpha=0.05$.

Les résultats du test montrent qu'il existe des différences statistiquement significatives dans les notes obtenues par les étudiants pour la deuxième partie du test (pour les cognates). Il n'y a pas de différence statistique dans les notes obtenues pour la première et la troisième partie du test. La note moyenne obtenue par le groupe A1 pour la 1-ère partie du test est de 15,5 points (sur 20), écart-type est de 2,23; la note moyenne obtenue par le groupe A2 pour la 1-ère partie du test-16,4 points (sur 20), écart-type est de 2,03, $\mathrm{T}_{1}$ (observé) $=1,317$. La note moyenne obtenue par le groupe A1 pour la partie 3 du test - 11,9 points (sur 20), écart type-2,08; note moyenne obtenue par le groupe A2 pour la partie 3 du test - 10,8 points (sur 20 ), écart type $1,98, \mathrm{~T}_{3}$ (observé) $=1,313$. Étant donné que le $\mathrm{T}$ observé dans les deux cas est inférieur à $\mathrm{T}$ critique $(\mathrm{T}$ crit. $(\mathrm{DF}=38 ; \alpha=0.05)=2,0243)$, il n'y a pas de différence 
statistiquement significative entre les notes moyennes des deux groupes au niveau d'importance standard 0.05 .

La note moyenne du groupe A1 pour la partie 2 du test-12,3 points (sur 20 ), écarttype-2,12; la note moyenne obtenue par le groupe A2 pour la partie 2 du test-15,3 points (sur 20 ), écart-type $2,17, \mathrm{~T}_{2}$ (observé ) $=4,570$. Étant donné que le T observé est supérieur au $\mathrm{T}$ critique (2,0243), il y a des différences statistiquement significatives entre les estimations moyennes des deux groupes (ayant le niveau standard d'importance 0.05 ). Les notes moyennes du groupe A1 (le français est LE 1) sont considérablement inférieures à celles du groupe A 2 (première langue est la langue orientale). Autrement dit, il existe une différence statistiquement importante entre les groupes en fonction de la première langue étrangère.

Le fait que les résultats des deux groupes pour la première partie du test sont meilleurs que ceux pour la troisième partie suggère que les sigles et les noms des organisations internationales ne présentent pas de problèmes lors de l'apprentissage, par des élèves, dans les conditions de contact linguistique, par opposition aux termes-expressions ("N $+\mathrm{A}^{\prime \prime}$. Les raisons de ce phénomène sont le transfert positif des noms des organisations internationales de la langue russe, de plus les étudiants suivent des cours de spécialité en langue maternelle en même temps que des cours de la "langue étrangère".

En ce qui concerne les termes formés par le modèle «nom + adjectif», la fréquence élevée des erreurs identifiées peut être due, y compris, l'interférence intra-linguistique («supergénération») dans la langue anglaise, à savoir, le transfert et le developpement de la règle de la préposition de l'adjectif devant le nom en anglais - le cas de la terminologie diplomatique. Le phénomène de surgénération explique le fait que les erreurs de cette partie du test sont faites par les étudiants, quel que soit leur LE 1.

\subsection{Etape 2}

\subsubsection{Contrôle de l'efficacité de la méthode d'apprentissage du vocabulaire professionnel}

Étant donné que les erreurs lexicales typiques des étudiants qui apprennent l'anglais comme LE 2 diffèrent et dépendent de la LE 1, il est important d'élaborer une méthode spéciale d'enseignement de l'anglais comme LE 2 si le francais la LE1, compléée de manuels et exercices appropriés. La méthode nécessaire a été élaborée et proposée par l'auteur.

Pour vérifier l'utilité et l'efficacité de la méthodologie proposée, les étudiants qui apprennent l'anglais comme 2-ème langue étrangère dans le cadre du cours "Anglais de la communication professionnelle" et le français comme LE1 ont été divisés en deux groupes homogènes (groupe B1 et groupe B2). Chaque groupe se composait de 20 étudiants. Pour le groupe-pilote on utilisait la méthode basée sur l' ensemble intégré d'exercices lexicaux visé à l'apprentissage de la lecture utilisant les méthodes de pédagogie critique; pour le groupe de controle on utilisant une méthode traditionnelle. Les deux groupes apprenaient la même discipline «Anglais de la communication professionnelle", ayant les mêmes buts et objectifs, le même manuel, travaillaient sous la direction d'un même enseignant. Les groupes ont été sélectionnés sur la base des questionnaires, des entretiens et des résultats des tests linguistiques / examens internationaux).

Le cours de l'anglais dans le domaine de la communication professionnelle a duré un semestre, deux heures par semaine. La performance académique a été effectuée au cours du contrôle intermédiaire et finale.

Au cours de l'enseignement, les entretiens et les questionnaires ont été proposés pour les étudiants afin de déterminer la progression de leurs connaissances du vocabulaire professionnel et les problèmes apparus à ce niveau. 
A la fin du cours, les étudiants des deux groupes ont été testés (60 questions) pour déterminer le niveau de la maîtrise du vocabulaire professionnel.

Les résultats du test sont présentés dans le tableau 2.

\begin{tabular}{|c|c|c|c|c|c|c|}
\hline & Groupe & $\mathrm{N}$ & Moyen & $\begin{array}{l}\text { Std. } \\
\text { Deviation }\end{array}$ & $\mathrm{DF}$ & $\begin{array}{l}\mathrm{T} \\
\text { observe }\end{array}$ \\
\hline \multirow[t]{2}{*}{$\begin{array}{l}\text { Partie 1. (Nom } \\
\text { des OI) }\end{array}$} & $\begin{array}{l}\text { Groupe } \\
\text { B1 }\end{array}$ & 20 & 18,3 & 1,65 & 38 & 2,286 \\
\hline & $\begin{array}{l}\text { Groupe } \\
\text { B2 }\end{array}$ & 20 & 17,1 & 1,67 & 38 & \\
\hline \multirow[t]{2}{*}{$\begin{array}{ll}\text { Partie } & 2 . \\
(\text { cognates }) & \end{array}$} & $\begin{array}{l}\text { Groupe } \\
\text { B1 }\end{array}$ & & 17,4 & 1,92 & 38 & 3,818 \\
\hline & $\begin{array}{l}\text { Groupe } \\
\text { B2 }\end{array}$ & & 15,9 & 2,01 & 38 & \\
\hline \multirow{2}{*}{$\begin{array}{ll}\text { Partie } & 3 . \\
\text { (expressions } \\
\text { "N+A") }\end{array}$} & $\begin{array}{l}\text { Groupe } \\
\text { B1 }\end{array}$ & & 16,7 & 2,11 & 38 & 2,413 \\
\hline & $\begin{array}{l}\text { Groupe } \\
\text { B2 }\end{array}$ & & 14,2 & 2,03 & 38 & \\
\hline
\end{tabular}

\section{Tableau 2: Résultats du test de vocabulaire professionnel des étudiants du groupe expérimental et celui de contrôle}

Le tableau 2 présente les résultats de l'application des statistiques $\mathrm{T}$ aux valeurs moyennes des notes du test obtenues par les étudiants du groupe B1 et B2 pendant le teste de différentes parties du texte. La possibilité d'appliquer le critère $\mathrm{T}$ de l'étudiant est due à l'homogénéité des variances, qui a été confirmée dans tous les cas par l'application des statistiques F, écarts-types avec un niveau d'importance standard de 0.05

La note moyenne obtenue par le groupe B1 pour la 1-ère partie du test-18,3 points (sur 20 ), écart-type-1,65; la note moyenne obtenue par le groupe B2 pour la 1-ère partie du test-17,1 points (sur 20), écart-type 1,67, T1 (observ.) = 2,286. La note moyenne obtenue par le groupe B1 pour la partie 2 du test-17,4 points (sur 20), écarttype-1,92; la note moyenne obtenue par le groupe B2 pour la partie 2 du test-15,9 points (sur 20 ), écart-type 2,01, T2 (observ.) = 3,818. La note moyenne obtenue par le groupe B1 pour la partie 3 du test-16,7 points (sur 20), écart-type-2,11; la note moyenne obtenue par le groupe B2 pour la partie 3 du test-14,2 points (sur 20), écarttype 2,03, T3 (observ.) $=2,413$. Comme le $\mathrm{T}$ observé dans les trois cas est plus superieur que le $\mathrm{T}$ critique ( $\mathrm{T}$ crit. $(\mathrm{DF}=38 ; \alpha=0.05)=2,0243)$, entre les notes moyennes obtenues à la suite du test, les deux groupes ont des différences statistiquement significatives ayant le niveau d'importance/ signification standard 0.05. Les notes moyennes du groupe pilote B1 sont nettement plus élevées que les celles du groupe de contrôle B2. Autrement dit, la distinction statistiquement significative a été trouvée entre les groupes à la suite de l'application d'une technique combinant l'utilisation d'un ensemble d'exercices intégratifs pour l'apprentissage du vocabulaire dans le processus de lecture intensive avec la technique de la pédagogie critique. Le test a révélé les différences statistiquement impotrantes/significatives entre les notes des groupes- pilotes et de contrôle pour les trois parties du test (tableau 2).

Il y a une différence considérable au niveau de la compétence lexicale : la note moyenne du groupe pilote / expérimental pour toutes les parties du test était plus élevée que celle du contrôle.

XLinguae, Volume 12 Issue 1XL, January 2019, ISSN 1337-8384, eISSN 2453-711X 
$\mathrm{Au}$ cours de l'enseignement, les étudiants du groupe expérimental ont souligné le développement et le perfectionnement des compétences en lecture et en compréhension du texte, l'enrichissement du vocabulaire, la motivation accrue et des compétences d'équipe.

Les résultats du contrôle intermédiaire et finale soulignent le développement des compétences lexicales dans le groupe expérimental.

Ainsi, on peut conclure que l'application de la méthode expérimentale élaborée par l'auteur a amélioré l'efficacité de la maîtrise du vocabulaire professionnel par les étudiants apprenant le français comme première langue étrangère.

\section{Conclusion}

Les résultats de l'étude confirment l'hypothèse de l'existence et de l'importance élevée de l'interférence linguistique dans l'étude de l'anglais professionnel en tant que deuxième langue étrangère et le français comme première langue étrangère; la source de l'interférence est le français. Les erreurs courantes des apprenants dépendent de leur expérience linguistique et sont dues à l'interférence linguistique de LE1 et LE2.

La source principale des erreurs est due aux cognates entièrement ou partiellement assimilés.

Les recherches ont abouti à la conclusion suivante: il est souhaitable de développer le complexe pédagogique spécial pour l'enseignement de la deuxième langue étrangère et proposer la variante qui combine l'utilisation d'un ensemble d'exercices intégratifs pour l'enseignement du vocabulaire dans le processus de lecture intensive avec la technique de la pédagogie critique.

L'expérience a confirmé l'utilité et l'efficacité de la méthodologie proposée en termes de formation des compétences lexicales, des compétences en lecture intensive, de développement du vocabulaire et de réduction des erreurs d'interférence linguistique.

Les résultats montrent que l'utilisation d'un ensemble d'exercices intégratifs pour l'apprentissage du vocabulaire dans le processus de lecture intensive en utilisant la méthodologie de la pédagogie critique contribue à la formation des compétences lexicales. Les données qualitatives et quantitatives de la recherche témoignent le fait que l'application de la méthodologie développée parallèlement avec les techniques de pédagogie critique a un effet positif sur l'assimilation du vocabulaire, développe les compétences en lecture intensive et enrichie le vocabulaire des apprenants, réduisant l'interférence linguistique lexicale et des erreurs typiques.

Les résultats obtenus peuvent être appliqués pendant la recherche ultérieure des phénomène de l'interférence lexicale pour développer des méthodes de formation des compétences lexicales lors de l'apprentissage de la deuxième langue étrangère, pendant les cours de l'anglais professionnel, ainsi que pendant l'élaboration de manuels spéciaux et des programmes de l'enseignement d'une deuxième langue étrangère.

\section{Remerciement}

Le travail a été effectué avec le soutien financier du ministère de l'éducation et de la science de la Fédération de Russie pour le Programme d'amélioration de la compétitivité du RUDN "5-100" parmi les principaux centres scientifiques et éducatifs du monde 2016-2020.

\section{Bibliographic references}

ALIKINA, O.V. Teaching the Vocabulary of French as a Second Foreign Language in the Process of Reading: Grades 8-9. Ph.D. in Pedagogy, 2009. 214 p.

BELLO, YEKEEN - OGUNDELE, M.O. - AGBOOLA, F.M. - AKANDE, E. I. Teaching and Learning of English Language by the French University Students in Porto-Novo, Benin Republic Asia. In: Pacific Journal of Education, Arts and 
Sciences. Vol. 2. No. 1. January, 2015. P-ISSN 2362-802; E-ISSN 2362-8030. Available online: www.apjeas.apjmr.com

BERRIDGE, G.R. - JONES, A. 2003. A Dictionary of Diplomacy. New York: McMillam. 296 p. ISBN-13: 978-1403915368.

CHOROLEEVA, K. 2009. Language Transfer Types of Linguistic Errors Committed by Francophones Learning English as a Second Foreign Language. In: Humanizing Language Teaching Magazine, pp. 90-102. Available online: http://www.hltmag.co.uk/oct09/.

CORDER, S.P. 1973. Introducing Applied Linguistics. Baltimore: Penguin Education. 392 p. ISBN $014071051 X$.

COUNCIL OF EUROPE. 2011. Common European Framework of Reference for Languages: Learning, Teaching, Assessment. Council of Europe. Cambridge: Cambridge University Press. 260 p. ISBN 0521803 136; ISBN 0521005310.

DUONG, THI QUYNH NGA. 2012. Réflexions sur les interférences dues au contact des langues en expression orale en français chez les étudiants d'anglais à l'Institut Polytechnique de Hanoï. In: Synergies Pays Riverains du Mékong. n.4 - 2012. pp. 13-25. ISSN : $2107-6758$.

ELLIS, R. 1986. Understanding Second Language Acquisition. Oxford: Oxford Univ. Press, 2nd, improved edition. $327 \mathrm{p}$.

FUSUN, SAVLI. 2009. Interférences lexicales entre deux langues étrangères: anglais et français. In: Synergies Turquie. n. 2. pp. 179-184. ISSN:1961-9472.

GAIRNS, R. - REDMAN, S. 1998. Working with Words: a Guide to Teaching and Learning Vocabulary. Cambridge University Press, 200 p. ISBN-13: 978-052131709; ISBN-10: 0521317096.

GASS, S. M. - SELINKER, L. 1994. Second Language Acquisition: an Introductory Course. (Topics in Applied Psycholinguistics.) Hillsdale, NJ: Lawrence Erlbaum, 357 p. ISBN 0-203-93284-6.

HANAFI, A. 2015. The Second Language Influence on Foreign Language Learners' Errors: The Case of the French Language for Algerian Students Learning English as a Foreign Language. In: European Scientific Journal (ESJ). V.15, issue 3. ISSN: 1857 7881. doi: 10.19044/esj.2014.v10n10p\%25p

KUHN, S. 2007. Interference of L1 English in L2 French Lexical Processing. Syracuse University Honors Program Capstone Projects. Paper 562.

LeBLANC, R. - SEGUIN, H. 1996. Les congeneres homographes et parographes anglais-francais. In: R. Courchêne, S. Burger, C. Cornaire, R. LeBlanc, S. Paribakht, $\&$ H. Séguin (Eds.), Twenty-five years of second language teaching at the University of Ottawa, pp. 69-91. Ottawa, Canada: University of Ottawa.

MAXIMOVA, O. 2016. Critical Pedagogy Approach to Improve Foreign Language Reading Skills / Ed. by L. Gomez Chova, A. López Martínez, I. Candel Torres // EDULEARN16 Proceedings. 8th International Conference on Education and New Learning Technologies. 2016. Barcelona: IATED Academy. pp. 8989-8995. ISBN: 978-84-608-8860-4. ISSN: 2340-1117. doi: 10.21125/edulearn.2016.0957

MAXIMOVA, O. 2016. Teaching Academic Reading in a Foreign Language: Meeting the Challenges of a Knowledge-based Society // 3rd International Multidisciplinary Scientific Conference on Social Sciences \& Arts SGEM 2016. Sofia: STEF92 Technology Ltd. P.1003-1010. ISBN-10: 978-619-7105-72-8. ISSN 2367-5669. doi 10.5593/sgemsocial2016B13

NATION, P. 2001. Learning Vocabulary in another Language. Cambridge: Cambridge University Press. 779 p. ISBN: 9781139524759. doi: $10.1017 /$ CBO9781139524759

NATION, P. 2004. Intensive Reading. EA Journal, Vol.21. no 2, pp. 20 - 29.

RICHARDS, J. C., (Ed) 1992. Error Analysis Perspectives on Second Language Acquisition. London: Longman. 228 p.

XLinguae, Volume 12 Issue 1XL, January 2019, ISSN 1337-8384, eISSN 2453-711X 
RITCHIE, W. C. - BHATIA, T. J. 1996. Handbook of Second Language Acquisition. San Diego: Academic Press. 758 p. ISBN 0125890427 ; ISBN 9780125890427.

SCHMITT, N. 2000. Vocabulary in Language Teaching. Cambridge: Cambridge University Press, 224 p. ISBN 0521 660483; ISBN 0521669383.

SUNDERMAN, G. - KROLL, J. F. 2006. First Language Activation During Second Language Lexical Processing: An Investigation of Lexical Form, Meaning, and Grammatical Class. In: Studies in Second Language Acquisition. Vol. 28. Issue 3 (September). pp. 387-422. doi:10.1017/S0272263106060177

THORNBURY, S. 2002. How to Teach Vocabulary. GB: Pearson Longman, 185 p. ISBN: 978-0-582-42966-6.

VERBITSKAYA, L. Globalization, Internationalization, and Language Learning / Russian Language Journal / Russkiy yazik. Vol. 55, No. 180/182 (2001-2005), pp. 715. ISSN: 00360252.

WALLACE, M. Practical Language Teaching, Teaching Vocabulary. London, Exeter, NH : Heinemann Educational Books, 1988. 144 p. ISBN: 0435289748.

WEINREICH, U. 1953. Languages in Contact. New York: Publications of the Linguistic Circle of New York, 148 p.

Words: 7486

Characters: 51135 (28,41 standard pages)

Assoc. prof. Maximova Olga

Department of Foreign Languages

Faculty of Humanities and Social Sciences

Peoples' Friendship University of Russia

6 Miklukho-Maklaya Street,

117198, Moscow

Russia

maximova-ob@rudn.ru

Assoc. prof. Spynu Larisa

Department of Foreign Languages

Faculty of Humanities and Social Sciences

Peoples' Friendship University of Russia

6 Miklukho-Maklaya Street,

117198, Moscow

Russia

spynu-1m@rudn.ru 